\title{
Author Index, Vol. 72, 1996
}

This index does not contain organizers and participants of Chromosome Mapping Workshops $1,3,10$ and 16 which are published in this volume.

Adelaide, J. 63 Agid,Y. 20 Alonso, M.L. 43 Andréo,B. 34 Apiou, F. 50 Avivi, L. 316 Ayraud, N. 171

Baker,E. 60 Banks, A. 250 Bar-Am, I. 316 Barcelo, G. 171 Barros, R.M.S. 325, 331 Beck,S. 299 Bellanné-Chantelot,

C. 5 Berg, A. van den 225 Bignon,C. 342 Birnbaum, D. 63 Blin,N. 299 Boreham,D.R. 179

Bouche, P. 20 Bowden, G.T. 179 Boxtel, J.A.F. van

303 Brice,A. 20 Brothman, A.R. 95 Buchwald,M. 194 Buys, C.H.C.M. 225

Calabrese, G. 155 Callen,D.F. 294 Cann,H.M. 5 Chaffanet,M. 63 Charlieu, J.P. 34 Chase, P.B. 205 Cimini,D. 219 Cohen, D. 5 Coignet, L. 34 Collins, E.E. 319 Combemale, P. 171 Cooper, D.W. 197 Corkery,B.M. 56 Cornwell,D. 37 Cowell,J.K. 72 Cupelli,L. 250

Dasouki,M. 252 Deen,P.M.T. 303 Degrassi, F. 219 De Meurichy, W. 310 Dorosz, S.D. 294

DuPont,B.R. 159 Dusetti,N. 83 Dutrillaux, B. 9,50

Echevarria, M. 303 Egashira, M. 175 Egozcue, J. 164 Eldridge, M.D.B. 197 Elsen,J.-M. 212

Endo, A. 46 Eyre,HJ. 294

Francke, U. 187 Freeman, M.L. 252 Fromaget, C. 185 Fujiwara,T. 86,191,

208, 242 Fukushima, Y. 78 Furutani, Y. 322

Garcia, D. 90

Geurts van Kessel, A.

303 Giorgi,D. 215 Girardet, A. 34 Glover,T.W. 319 Gött,P. 299 Göttert, E. 40 Goubin,G 342

Gouider, R. 20 Grant, A.L. 319 Grifo,J. 43 Gros,D. 185 Guerini, D. 236 Gugenheim, M. 20

Gunn,H.C. 294

Halonen,M. 205 Hawthorn, L.A. 72 Hayes, H. 342 Heine-Suñer, D. 339 Held,M. 246

Heng,H.HQ. 217 Hirai,Y. 208 Hirano,H. 86 Hirata, T.-I. 183 Hitomi, Y. 12 Holmes, GH. 306

Horowitz, M. 316 Hoshikawa, H. 322 Huang, W.-Y. 217 Hulsbeek, M.M.F. 225

Ikura, T. 322 Imbert, A. 63 Inazawa, J. 78 lovanna, J. 83 Ishikawa, H. 46

Jinno,Y. 177 Johnson, V.P. 95 Johnston, P.G. 197 Jong, D. de 225

Kaneda,Y. 183 Kanetake, H. 177 Kas,K. 297 Kawai,A. 86,208, 242 Kawakura, K. 183 Kitamura, D. 175 Klee, C.B. 236 Knight, C.B. 159 Knox,J.D. 179

Kohno,K. 342 Kok,K. 225

Kondoh,N. 12 Kondoh,S. 183 Kooy,R.F. 225 Koyama, K. 78 Krauter,K. 250 Krishnamani, M.R.S.

252 Kubota,Y. 12 Kucherlapati, R.S.

250

Laing,N.G. 294 Lanneluc, I. 212 LaStarza, R. 229 Laurent, A.-M. 69 Law,D.J. 319 Leach, R.J. 90,159 Leblanc-Straceski, J. 
250 LeChalony,C. 342 Lefort,G. 34 LeGuern, E. 20 Lemieux, N. 9 LePaslier, D. 63

Leppig,K.A. 95 Liebaers, I. 53 Liew,C.-C. 217 Lim,L. 294 Linn,R. 90, 159 Lissens, W. 53

Little, M.H. 306 Lombard, M. 9 Long, S.E. 162 Lopes, J. 20

McBride,O.W. 236 McConkey, E.H. 60 McKenzie, L.M. 197 Márquez, C. 164 Martin-DeLeon, P.A.

56 Martorell, M.R. 164 Marynen,P. 229,310 Masaki, T. 322 Mastroprimiano, G

155 Matrisian, L.M. 179 Matsuda, Y. 1 Matsumoto,N. 177 Matsuoka,R. 322 Mattei,M.-G 185

Mattevi,M.S. 325,

331 Mecucci, C. 229 Meese, E. 40 Menon, R. 60 Mercier, D. 342 Meredith, M. 252 Mesnil,M.

185 Meyen,E. 297 Meyer,J. 246 Mignon,C. 185 Miller,D.E. 319 Minoletti,F. 171

Miyake,Y.-I. 183 Morielle-Versute, E.

26 Mohrer,M. 90 Moker,J.S. 37 Mongelard, F. 200 Moníer,K. 200 Moore, S. 90 Moreno, V. 164

Morrison, D.P. 179 Mueller-Lantzsch, N.

40 Mulders, S.M. 303 Müller,C.R. 215 Mulsant,P. 212 Muniz, J.A.P.C. 331 Munné, S. 43

Murakami, R.-K. 183

Nagamachi, C.Y.

325,331 Nagata,M. 86,208,

242 Nagle,R.B. 179 Nakamura, Y. 78, 86,

191,208,242 Naylor,S.L. 90 Negus, K. 306 Neil, S. 95 Nemani, M. 5 Niikawa,N. 175, 177

O’Connell,P. 90 Oikawa, T. 12 Okui,K. 191 Okuno,S. 86,242 Olde Weghuis, D. 303 Os, C.H.

van 303 Ozaki,K. 86,242

Palka,G 155 Pardo-Manuel de

Villena,F. 339 Payen,E. 342 Pébusque, M.-J. 63 Pedeutour, F. 171 Peila,R. 155 Pellestor,F. 34

Petit, P. 310 Petty, E.M. 319 Phillips, J.A., III 252 Pieczarka, J.C. 325,

331 Plaetke,R. 159 Prades, C. 69 Puechberty, J. 69

Quenesson, I. 34

Rao,P.N. 252 Ravise,N. 20 Regan, J.W. 205 Renault,B. 250 Reus,B. 90

Robert-Nicoud, M.

200 Rodriguez de

Cordoba, S. 339 Roizès, G. 69 Rouquier, S. 215 Rubenstein, A. 95 Rumpler,Y. 50

Saidi-Mehtar, N. 212 Saito,H. 191 Saito,Y. 177 Sakai,H. 177 Sakurada, K. 46 Sakurai, S. 1

Sasaki, T. 177 Sauter, M. 40 Sawamura, T. 322 Schanen,N.C. 187 Schempp, W. 246 Scherer, G. 246 Scherer, S.W. 187 Schmitt,H. 299 Schmutz, S.M. 37 Schommer, S. 40 Schwarz, M. 331

Shimada, K. 322 Shimada,Y. 86,242 Shimizu,F. 86,208,

242 Shin,S. 86, 191,242 Shiraki,T. 322 Shizuya,H. 299 Sierra-Rivera, E. 252 Simon, M.I. 299

Simon, M.P. 171 Sly,W.S. 53 Sood,S. 194 Sozzi,G. 171 Speleman,F. 53, 189 Stanton,V. 90

Stavropoulos, D.J.

194 Stephanova, E. 83 Stephens, K. 95 Stuppia, L. 155 Summar, M.L. 252 Sutherland, G.R. 60

Suzuki, H. 1 Szepetowski, P. 200 Szpirer, C. 83 Szpirer, J. 83

Taddei, V.A. 26 Takahashi, E. 86,

208, 242 Takahashi, N. 78 Takaichi,A. 86,242 Takami, S. 86,242 Takeda,S. 86,242 Tanzarella, C. 219 Tanzawa, K. 322 Templado,C. 164 Tenaglia, R. 155

Terrier-Lacombe,

M.J. 171 Thompson, F.H. 205 Tihy,F. 9 Tissir, F. 83 Tomkins,D.J. 194 Tomotsune, D. 78

Troyer, D.L. 37 Tsui,L.-C. 187 Turc-Carel, C. 171

Uchiumi,F. 12 Usson,Y. 200 
Van Camp, G. 189 Van den Berghe, H.

229,297,310 Van de Ven, W.J.M.

297 Van Roy, N. 53, 189 Varella-Garcia, M. 26 Veen, A.Y. van der

225 Vermeesh, J.R. 310 Vervoort, R. 53 Vezuli,A. 50 Viskochil, D. 95 Vourc'h,C. 200

Wagner, M.J. 63 Wainwright, B.J. 306 Walker, J.-A. 179 Wang, M.G. 236 Ward,D. 250

Warter,S. 50 Watanabe,T. 175,242 Watanabe, T.K. 86,

208 Wells, D.E. 63 Welter,C. 299 Wendelmuth, U. 215 Wicking,C. 306 Williams, G. 60

Wilton, S.D. 294 Wirth, J. 246 Wlodarska,I. 229,

297 Wolz,W. 215 Wundrack,I. 299

Xiang,X. 90 Xin,X. 90

Yamada,K. 322 Yamada, T. 12 Yamamoto, T. 12 Yamasaki,H. 185 Yang,J.-M. 205 Yi,H. 236

Yoshida,M. 322 Yurov,Y. 69

Zang,K,D. 40 Zhu,X.L. 95

347 